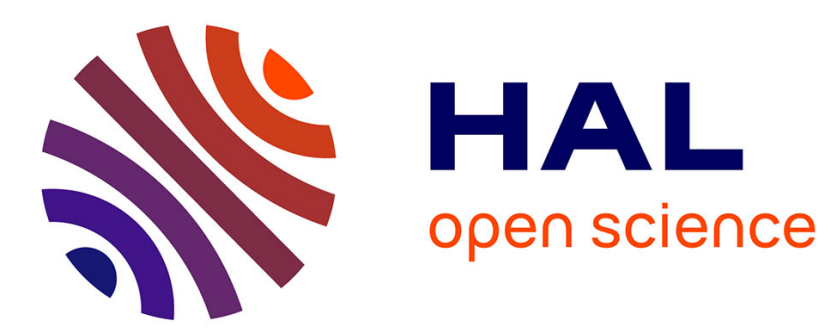

\title{
Energy-based variational modeling of fully formed adiabatic shear bands
}

Shaopu Su, Laurent Stainier, Sébastien Mercier

\section{To cite this version:}

Shaopu Su, Laurent Stainier, Sébastien Mercier. Energy-based variational modeling of fully formed adiabatic shear bands. European Journal of Mechanics - A/Solids, 2014, 47, pp.1-13. 10.1016/j.euromechsol.2014.02.010 . hal-00983537

\section{HAL Id: hal-00983537 https://hal.science/hal-00983537}

Submitted on 6 Nov 2018

HAL is a multi-disciplinary open access archive for the deposit and dissemination of scientific research documents, whether they are published or not. The documents may come from teaching and research institutions in France or abroad, or from public or private research centers.
L'archive ouverte pluridisciplinaire HAL, est destinée au dépôt et à la diffusion de documents scientifiques de niveau recherche, publiés ou non, émanant des établissements d'enseignement et de recherche français ou étrangers, des laboratoires publics ou privés. 


\title{
Energy-based variational modeling of fully formed adiabatic shear bands
}

\author{
Shaopu Su ${ }^{a}$, Laurent Stainier ${ }^{\mathrm{a}}$, Sébastien Mercier $^{\mathrm{b}}$ \\ ${ }^{a}$ Institut de Recherche en Génie Civil et Mécanique (GeM - UMR 6183 CNRS) Ecole Centrale de Nantes, 1 rue de la Noë, BP 92101, F-44321 Nantes, France \\ ${ }^{\mathrm{b}}$ Laboratoire d'Etude des Microstructures et de Mécanique des Matériaux, Université de Lorraine, CNRS UMR 7239, Ile du Saulcy, F-57045 Metz, France
}

\begin{abstract}
An energy-based variational modeling approach of adiabatic shear bands (ASB) for thermo-viscoplastic materials is proposed. In the process of derivation of this thermo-mechanical coupled problem, the variational methodology is first applied to classical problems, such as Couette flow, thermal conduction in a fluid layer and thermal Couette flow. The solutions are compared to the corresponding analytical solutions. In a second part, a slab under stationary simple shear is analyzed by a Ritz-Galerkin method combined with an energy-based variational approach of thermo-mechanical problems. The velocity and temperature profiles within an ASB are parameterized by two quantities: the shear band width and central temperature. It is shown that this variational formulation works for various material constitutive models, as illustrated by adopting two popular constitutive models (power law and Johnson-Cook law) and the results are checked by using a variational Finite Element Method. In a last part, mixed heat exchange boundary conditions are introduced to limit the effect of slab width on the solution. The profiles of velocity and temperature in the slab, as well as the influence of material properties on the formation of shear band are evaluated and in good agreement with results from the literature.
\end{abstract}

\section{Introduction}

Adiabatic shear bands (ASB) are generally considered as resulting from a thermo-mechanical instability leading to large deformation and high temperature in a narrow band, typically a few tens of micrometers in width (Zener and Hollomon, 1944; Merzer, 1982; Wright, 2002). The development of ASB is assumed to proceed in three steps: in stage I, the deformation in shear is homogeneous, and strain hardening of the material overcomes thermal softening effects; in stage II, after the maximum stress has been reached, a diffuse instability develops due to the prominence of thermal softening, and the deformation begins to be slightly heterogeneous; in stage III, a strong instability develops and deformation localizes in a narrow band. Fracture may occur in this third stage. The instability can be triggered by a geometrical imperfection, or by an heterogeneity, either in temperature, stress, or microstructure. Note that other softening mechanisms than temperature can be at play: in
Keywords:

Adiabatic shear band

Variational approach

Thermo-viscoplasticity particular, recent work (Rittel et al., 2008; Dolinski et al., 2010) has shown the important role of micro-mechanisms such as dynamic recrystallization, which could also be at the origin of ASB formation. In the present paper, we will nonetheless restrict ourselves to thermal softening. Under certain circumstances, when the hardening is saturated and the heat produced by visco-plastic dissipation is removed by heat conduction, a steady state may develop in the final stage. In the following, the fully developed adiabatic shear band under steady state is analyzed, setting aside early stages of formation.

Because of their narrow width and inhomogeneous temperature distribution, it is very difficult to experimentally capture detailed features of ASBs, such as central temperature. Consequently, a large part of the literature concentrates on theoretical and numerical simulations. Numerous contributions propose a one-dimensional analysis of ASB (canonical problem) to capture the critical condition for occurrence of ASB and the velocity profile inside the band (Merzer, 1982; Leroy and Molinari, 1992; Wright and Ockendon, 1992; Wright and Ravichandran, 1997; DiLellio and Olmstead, 1997, 1998, 2003; Dinzart and Molinari, 1998). An interesting outcome of the cited papers is the crucial role of heat transfer (thermal conductivity) in structuring the shear localization. Twodimensional analyses of ASB were also considered to determine 
shear band propagation (Gioia and Ortiz, 1996; Mercier and Molinari, 1998). However, analytical works cannot easily be extended to general constitutive relations and real configurations (3D initiation, transient regime). These methods are thus not best adapted to study full complex applications.

Numerical simulation of ASB is difficult because of large deformation gradients and strong thermal softening existing during its development. Difficulties such as mesh dependence, interactions between multiple bands, require advanced numerical methods (Yang et al., 2005; Areias and Belytschko, 2007). To bypass these problems, some authors have proposed to introduce discontinuities to accurately describe the shear localization (Ortiz et al., 1987; Oliver et al., 1999). In most of these numerical approaches, it is necessary to know approximately the domain where the shear localization takes place. Predicting the width of shear band in thermo-viscoplastic materials is another prerequisite to proceed to the dynamic evolution of shear localization. Many challenges thus remain unsolved concerning shear localization. For example, this is why it is difficult to simulate forming operations and response of structures when ASBs are involved.

In this paper, starting from canonical solutions of velocity and temperature profiles (Leroy and Molinari, 1992), we propose a new method to define the key parameters of ASB (width, maximum temperature). This method relies on the variational formulation of coupled thermo-mechanical boundary-value problems recently proposed by Yang et al. (2006). This formulation allows to describe thermal and mechanical balance equations, including irreversible and dissipative behaviors, under the form of an optimization problem of an energy-like functional. The variational formulation can adopt a wide range of constitutive models and also presents interesting mathematical properties like symmetry, inherent to variational approaches, contrarily to alternative coupled thermomechanical formulations. Applying this formulation, Stainier and Ortiz (2010) were able to model the thermo-viscoplastic behaviors of materials such as Aluminum alloy, $\alpha$-Titanium and Tantalum.

As an application of this variational formulation, we aim at building an energy-based variational modeling to analyze shear localization in one dimension. This paper is organized as follows: the variational approach for the thermo-mechanical problem is simply restated. In the following section, we validate the theory by considering classical problems such as Couette flow, thermal conduction in a layer and non-Newtonian thermal-Couette flow. In Section 5, the fully formed adiabatic shear band is considered and the configuration proposed in Leroy and Molinari (1992) is first adopted. The thermo-mechanical coupled variational approach is used to predict the shear band width and central temperature in the adiabatic shear band. Exact results of Leroy and Molinari (1992) are retrieved. Meanwhile, effects of material parameters (thermal softening, strain-rate sensitivity) on these two features are analyzed. Then, different constitutive laws, power-law strain-rate dependency and thermal softening, and classical Johnson-Cook law, are adopted to demonstrate the versatility of the proposed approach. Since analytical solutions do not exist for general constitutive behaviors, a variational-based Finite Element Method (FEM) provides the evolutions of velocity and temperature up to the steady state. It is shown that the results obtained by the proposed variational model are in good agreement with FEM. Finally, we consider the introduction of a heat exchange thermal flux at remote boundaries of the layer so as to obtain invariance of the shear band width for different sizes of the layer.

\section{Variational framework}

In this section, the variational formulation of the coupled thermo-mechanical boundary-value problem proposed by Yang et al. (2006) is summarized. The potential of total power density is stated for general dissipative materials as follows:

$$
\begin{aligned}
\Phi[\dot{\boldsymbol{\varphi}}, T, \dot{N}, \dot{\mathbf{z}}]= & \int_{B_{0}}\left[\dot{E}-T \dot{N}+\Delta\left(\frac{T}{\Theta} \dot{\mathbf{F}}, \frac{T}{\Theta} \dot{\mathbf{Z}},-\frac{\nabla_{0} T}{T} ; \Theta\right)\right] \mathrm{d} V_{0} \\
& -\int_{B_{0}} \rho_{0} \mathbf{b} \cdot \dot{\boldsymbol{\varphi}} \mathrm{d} V_{0}-\int_{\partial_{T} B_{0}} \overline{\mathbf{T}} \cdot \dot{\boldsymbol{\varphi}} \mathrm{d} S_{0} \\
& +\int_{B_{0}} \rho_{0} Q \log \frac{T}{T_{0}} \mathrm{~d} V_{0}-\int_{\partial_{N} B_{0}} \bar{H} \log \frac{T}{T_{0}} \mathrm{~d} S_{0}
\end{aligned}
$$

where $B_{0}$ is the undeformed reference configuration, $\mathbf{b}$ is the body force per unit mass, $\rho_{0}$ is the mass density per unit undeformed volume, $\overline{\mathbf{T}}$ is the applied traction force over the traction boundary $\partial_{\mathrm{T}} B_{0}, Q$ and $\bar{H}$ are the specified heat source per unit mass and the outward normal heat flux over the Neumann boundary $\partial_{\mathrm{N}} B_{0}, \varphi$ is the displacement mapping, and $N=\rho_{0} \eta$ is the nominal entropy density ( $\eta$ being the entropy per unit mass). $T$ and $\Theta$ are respectively an external temperature and an equilibrium temperature. This distinction is introduced to ensure the symmetry of the variational formalism, although $T=\Theta$ in the local thermodynamic state. $E$ is the internal energy density (per unit undeformed volume), related to the Helmholtz free energy $W$ by a Legendre transform:

$E=\sup _{T}\left[\rho_{0} \eta T+W\right]$

Furthermore the equilibrium temperature is defined by:

$\Theta=\frac{\partial E}{\partial\left(\rho_{0} \eta\right)}$

The thermo-mechanical coupling originates from the thermal dependence of parameters involved in the plasticity constitutive relations and from the dissipation due to viscoelasticity and viscoplasticity parts. The general dissipation potential $\Delta$ is defined by:

$\Delta(\dot{\mathbf{F}}, \dot{\mathbf{Z}}, \mathbf{G} ; \Theta)=\phi^{*}(\dot{\mathbf{F}} ; \Theta)+\psi^{*}(\dot{\mathbf{Z}} ; \Theta)-\chi(\mathbf{G} ; \Theta)$

where $\psi^{*}, \phi^{*}$ and $\chi$ are respectively the kinetic potential, the viscous potential (Kelvin-Voigt viscoelasticity) and the conduction potential, and where $\mathbf{G}=-\nabla_{0} T / T$. In the above expressions, we have included the possibility of a parametric dependence on temperature $\Theta$. The first Piola-Kirchhoff stress $\mathbf{P}$ is the conjugate variable of the deformation gradient $\mathbf{F}=\nabla_{0} \varphi$ :

$\mathbf{P}=\frac{\partial W}{\partial \mathbf{F}}+\frac{\partial \phi^{*}}{\partial \dot{\mathbf{F}}}$

The driving forces $\mathbf{Y}$ and the internal variables $\mathbf{Z}$ are conjugate variables so that:

$\mathbf{Y}=-\frac{\partial W}{\partial \mathbf{Z}}=\frac{\partial \psi^{*}}{\partial \dot{\mathbf{Z}}}$

Finally, the nominal heat flux $\mathbf{H}$ is given by:

$\mathbf{H}=\frac{\partial \chi}{\partial \mathbf{G}}$

Choosing potentials $\psi^{*}, \phi^{*}$ and $\chi$ as non-negative and convex functions of their main argument (resp. $\dot{\mathbf{Z}}, \dot{\mathbf{F}}, \mathbf{G}$ ) will then be a sufficient condition for verifying the second law in thermodynamics. 
From the above description, the thermal and mechanical balance equations, the constitutive relations, as well as the equality between the external temperature and equilibrium temperature are the Euler-Lagrange equations of the following variational formulation:

$\inf _{\dot{\varphi}, \dot{\mathbf{Z}}, \dot{N}} \sup _{T} \Phi[\dot{\varphi}, T, \dot{N}, \dot{\mathbf{Z}}]$

The thermo-mechanical coupling for general dissipative materials can thus be described as an optimization problem, and many mathematical algorithms, such as trust-region method, Levenberg-Marquardt algorithm, are suitable to seek a minimum or maximum value with respect to physical fields. In contrast to conventional coupled thermo-mechanical problem formulation, this variational approach intrinsically yields a symmetric stiffness matrix when adopting finite element methods based on this variational framework. Indubitably, these characteristics allow the application of a broad range of mathematical algorithms, contributing to numerical efficiency in matrix storage and nonlinear programming. Furthermore, this variational formulation seamlessly works for general standard materials. Consequently in view of these advantages, variational principle (8) allows to design robust and efficient algorithms, such as the Ritz-Galerkin method that we will adopt within the present paper.

In the following, we restrict our attention to steady state problems ( $\dot{E}=\dot{N}=0$ ) and isotropic materials. An Eulerian description can thus be adopted, and the total power density potential reduces to:

$\Phi=\int_{B} \Delta\left(\frac{T}{\Theta} \boldsymbol{\nabla} \boldsymbol{V}, \frac{T}{\Theta} \dot{\mathbf{Z}},-\frac{\nabla T}{T} ; \Theta\right) \mathrm{d} V$

where body forces and heat sources, imposed surface tractions and heat fluxes have been neglected. The integral in (9) is now taken on the current deformed configuration (assumed to be stationary).

\section{Couette flow and thermal conduction in a layer}

As a first validation, we consider an isothermal Couette flow where two infinite plates, one of which subjected to a shearing velocity $V_{0}$ relatively to the other, are separated by a distance $L$. The viscous fluid located between the plates is considered Newtonian and incompressible, so that Navier-Stokes equation can be written as follows:

$\frac{\partial^{2} V}{\partial y^{2}}=0$

By using the boundary conditions:

$V(0)=0 ; \quad V(L)=V_{0} ;$

the analytical velocity can be written:

$V(y)=\frac{V_{0}}{L} y$

Under steady state and without thermal conduction, the potential $\Phi$ in (9) reduces, for the Couette flow, to

$\Phi=\int_{B} \phi^{*} \mathrm{~d} V$

For an incompressible and Newtonian fluid, $\phi^{*}=1 / 2 \mu\left(V_{y}\right)^{2}$, where $\mu$ represents the fluid viscosity. We adopt the following polynomial form to describe velocity fields:
$V(y)=\sum_{i=0}^{N} a_{i} y^{i}$

which is simplified through the boundary conditions as:

$\left.V(y)=\frac{V_{0}}{L}-\sum_{i=2}^{N} a_{i} L^{i-1}\right) y+\sum_{i=2}^{N} a_{i} y^{i}$

where $a_{i}(i=2, \ldots, N)$ are unknown parameters to be defined. Adopting the velocity field (15), variational principle (8) becomes:

$\inf _{a_{i}} \Phi(V)$

So defining the velocity of the Couette flow reduces to an optimization problem with respect to coefficients $a_{i}$. These coefficients can be calculated by the corresponding Euler-Lagrange equation of (16), yielding $a_{i}=0(i=2, \ldots, N)$. The analytical solution is thus retrieved.

Next, the variational principle is tested on a simple conduction problem. Consider a 1D layer (of width $L$ ) with fixed temperature boundary conditions:

$T(0)=T_{0} ; \quad T(L)=T_{1} ;$

In that case, the total power density is:

$\Phi=\int_{B}-\chi\left(-\frac{T_{, y}}{T} ; \Theta\right) \mathrm{d} V$ where $\chi=\frac{1}{2} \Theta \lambda\left(\frac{T_{, y}}{T}\right)^{2}$

where $\lambda$ is the thermal conductivity. As previously described, $\Theta$ is the equilibrium temperature introduced to satisfy the symmetry property of the variational formulation. Note that $\Theta=T$ in steady state. Hence the variational model of the thermal layer is written as

$\sup _{T} \Phi(T)$

Similarly to the foregoing Couette flow problem, we adopt the following trial temperature field which satisfies the boundary conditions:

$T(y)=T_{0}+\left(\frac{T_{1}-T_{0}}{L}-c L\right) y+c y^{2}$

Using Galerkin method, we recover the analytical solution, corresponding to $c=0$ :

$T(y)=T_{0}+\frac{T_{1}-T_{0}}{L} y \quad y \in[0, L]$

\section{Thermal Couette flow}

Merging the above two examples, the Couette flow with thermal effect is next considered. In Fig. 1, two plates at constant temperature $T_{0}$ and $T_{1}$ are considered. The bottom plate is fixed while the top plate is moving at constant velocity $V_{0}$.

In the present case, the fluid is considered incompressible and non-Newtonian. The constitutive model is given by:

$\tau=\mu \dot{\gamma}_{0}\left(\frac{\dot{\gamma}}{\dot{\gamma}_{0}}\right)^{m}$

where $m$ is a strain rate sensitivity exponent, $\tau$ is the effective shear stress, $\dot{\gamma}$ is the effective shear strain rate and $\dot{\gamma}_{0}$ is a reference strain rate. The conservation of mass, the conservation of linear momentum and the conservation of energy are simplified to: 


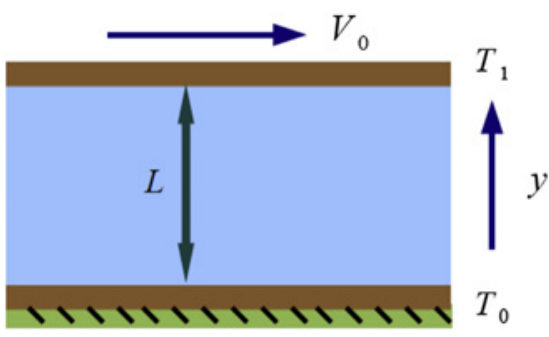

Fig. 1. 1D thermal Couette flow problem.

$V_{, x}=0 \quad \tau_{, y}=0 \quad p_{, y}=0 \quad-\lambda \frac{\partial^{2} T}{\partial y^{2}}=\tau \dot{\gamma}$

where $p$ represents the pressure and is uniform in our problem. Obviously, the analytical profiles of velocity and temperature are:

$V(y)=\frac{V_{0}}{L} y$,

$\begin{aligned} T(y)= & -\frac{1}{2} \frac{\mu}{\lambda \dot{\gamma}_{0}^{m-1}}\left(\frac{V_{0}}{L}\right)^{m+1} y^{2}+\left[\frac{T_{1}-T_{0}}{L}+\frac{1}{2} \frac{\mu}{\lambda \dot{\gamma}_{0}^{m-1}}\left(\frac{V_{0}}{L}\right)^{m+1} L\right] y \\ & +T_{0}\end{aligned}$

Considering the thermal Couette flow in steady state, the total power density has two contributions: the viscous potential and the conduction potential:

$\Phi(V, T)=\int_{0}^{L}\left[\frac{1}{m+1} \frac{\mu}{\dot{\gamma}_{0}^{m-1}}\left(\frac{T}{\Theta} V_{, y}\right)^{m+1}-\frac{1}{2} \Theta \lambda\left(\frac{T_{, y}}{T}\right)^{2}\right] \mathrm{d} y$

Note that expressing stationarity of $\Phi$ with respect to $V$ and $T$ leads to momentum and energy balance equations. The following trial velocity and temperature are adopted:

$V(y)=a y^{2}+\left(\frac{V_{0}}{L}-a L\right) y$

$T(y)=c y^{2}+\left(\frac{T_{1}-T_{0}}{L}-c L\right) y+T_{0}$

where $a$ and $c$ are the unknown parameters. The variational model of thermal Couette flow is restated and rewritten as:

$\inf _{a} \sup _{c} \Phi(V, T)$

Finding the solution of the thermal Couette flow turns to a mathematical optimization problem with a saddle point for the two unknowns $a$ and $c$. Adopting the material parameters listed in Table 1, values of $a$ and $c$ obtained from the analytical solutions and the variational modeling are given in Table 2. We obtain a perfect agreement (which was expected since our space of trial functions

Table 1

Material properties for thermal Couette flow problem.

\begin{tabular}{llllllll}
\hline$\mu(\mathrm{GPa} / \mathrm{s})$ & $\dot{\gamma}_{0}\left(\mathrm{~s}^{-1}\right)$ & $\lambda\left(\mathrm{Wm}^{-1} \mathrm{~K}^{-1}\right)$ & $V_{0}(\mathrm{~m} / \mathrm{s})$ & $m$ & $T_{0}(\mathrm{~K})$ & $L(\mathrm{~m})$ & $T_{1}(\mathrm{~K})$ \\
\hline 0.157 & 1 & 0.6 & 50 & 0.5 & 273 & 0.2 & 300 \\
\hline
\end{tabular}

includes the analytical solution). Fig. 2 presents the corresponding profiles of velocity and temperature in the layer. The velocity has a linear form with a slope $V_{0} / L$, while the temperature in the fluid between the plates is gradually increasing with a parabolic form from the cold plate to the hot plate.

\section{Fully formed adiabatic shear band}

The variational principle in continuum form has shown its efficiency in deriving exact solutions of classical problems. We consider next a fully coupled thermo-mechanical problem: the formation of ASB. As described in Fig. 3, a slab of thickness $2 \mathrm{H}$ is subject to simple shearing by imposing velocity $\pm V_{0}$ and isothermal conditions $T=T_{0}$ at $y= \pm H$. The material is representative of steel with a thermo-viscoplastic behavior as considered in Leroy and Molinari (1992). The constitutive relation is written as:

$\tau=\tau_{0} \exp \left[-\beta\left(\frac{T}{T_{0}}-1\right)\right]\left(\frac{\dot{\gamma}}{\dot{\gamma}_{0}}\right)^{m}$

Hardening is disregarded in this section (e.g. we consider a saturated hardening regime). Constitutive parameters $\tau_{0}$ and $\dot{\gamma}_{0}$ are reference stress and strain rate, while $T_{0}$ is a reference temperature, taken equal to the imposed temperature at $y= \pm H$. Coefficient $\beta$ defines the level of thermal softening and $m$ is the strain rate sensitivity. We consider that a steady state prevails so that the elastic strain rate and the entropy rate are zero. Under these conditions, the total strain rate is equal to the plastic one. For the proposed problem, the total power density then reduces to:

$\Phi(V, T)=\int_{-H}^{H}\left[\psi^{*}\left(\frac{T}{\Theta} V_{, y} ; \Theta\right)-\chi\left(-\frac{T_{y}}{T} ; \Theta\right)\right] \mathrm{d} y$

where the dissipation pseudo-potential $\psi^{*}$ is defined based on the flow stress relation of the material (28):

$\psi^{*}(\dot{\gamma} ; \Theta)=\frac{\tau_{0} \dot{\gamma}_{0}}{m+1} \exp \left[-\beta\left(\left(\frac{\Theta}{T_{0}}\right)-1\right)\right]\left(\frac{\dot{\gamma}}{\dot{\gamma}_{0}}\right)^{m+1} \quad m \in[0,1]$

The second part $\chi$ is the conduction potential corresponding to the isotropic Fourier law, given by (18). The problem in Fig. 3 can thus be solved by adopting the following optimization formulation:

$\inf _{V} \sup _{T} \Phi(V, T)$

The mechanical equilibrium equation derives from the stationarity condition on velocity $V$, while the heat equation derives from the stationarity condition on temperature $T$ (see Appendix A). In addition, thermal equilibrium requires that $\Theta=T$. Leroy and Molinari (1992) have shown that an exact solution exists for the problem of Fig. 3 when the material flow law is given by (28). In this section, HY100 steel with material parameters given in Table 3 is considered.

Table 2

Values of parameters $a$ and $c$ obtained from the analytical solutions and the variational method.

\begin{tabular}{lll}
\hline & Variational modeling & Analytical solutions \\
\hline$a\left(\mathrm{~m}^{-1} \mathrm{~s}^{-1}\right)$ & $-2.84 \times 10^{-13}$ & 0 \\
$c\left(\mathrm{Km}^{-2}\right)$ & -517.16 & -517.16 \\
\hline
\end{tabular}




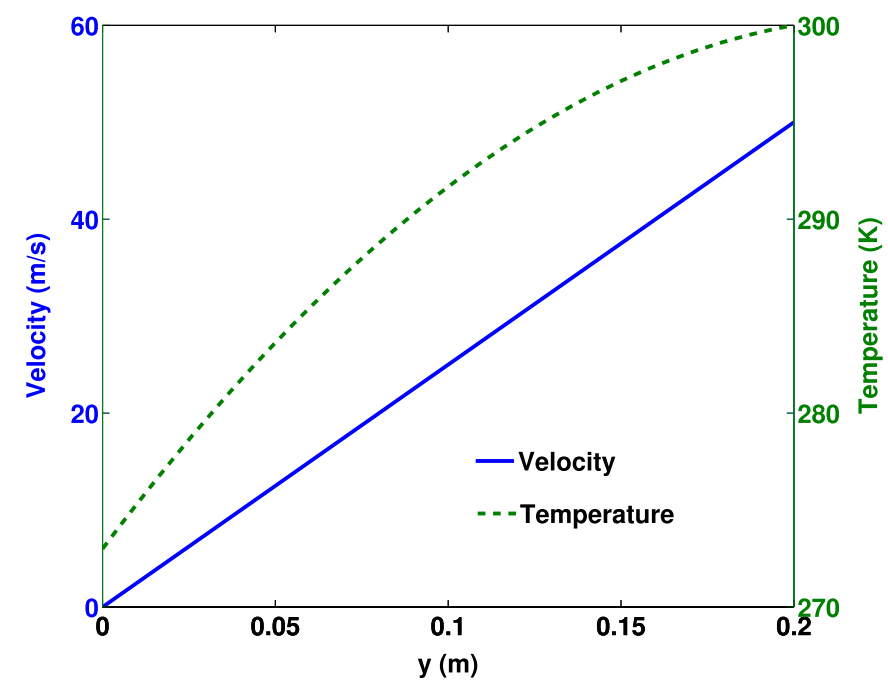

Fig. 2. Profiles of velocity and temperature in the thermal Couette flow.

\subsection{Ritz-Galerkin method}

As stated before, Leroy and Molinari (1992) gave analytical expressions for the profiles of velocity and temperature in a layer made of material whose behavior is modeled by (28) and with the following boundary conditions:

$\left.V\right|_{ \pm H}=V_{0} ;\left.\quad T\right|_{ \pm H}=T_{0}$

Wright and Ravichandran (1997) observed that these expressions were representing a good approximate for different constitutive models.

Our aim is to show that the proposed variational formulation is able to recover the exact solution when the material constitutive model is given by (28). In addition, it will be shown that this approach can also provide accurate results for other constitutive models.

\subsubsection{Shear band width and penalty method}

In this section, the trial functions representing the velocity and the temperature within the ASB are based on the analytical solutions derived by Leroy and Molinari (1992) and considered as canonical functions by Wright and Ravichandran (1997):

$V(y)=V_{0} \frac{\tanh \left(y / h_{V}\right)}{\tanh \left(H / h_{V}\right)}, \quad T(y)=T_{0}\left[1-\frac{2 m}{\beta} \ln \frac{\cosh \left(y / h_{T}\right)}{\cosh \left(H / h_{T}\right)}\right]$

Two length-like parameters $\left(h_{V}\right.$ and $h_{T}$ ) have been introduced. Owing to symmetry, the actual widths are twice these values.

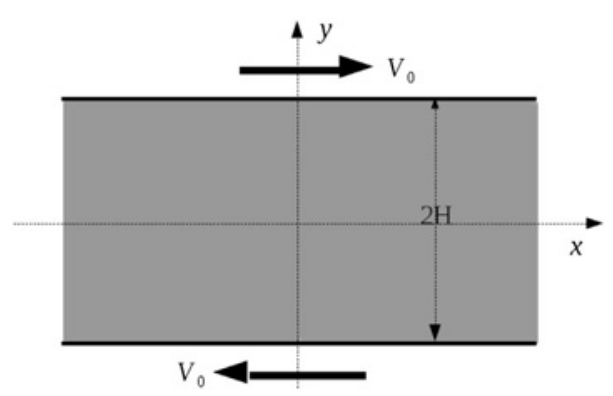

Fig. 3. 1D shear band configuration Leroy and Molinari (1992).
Table 3

Material properties for HY100 steel (Leroy and Molinari, 1992).

\begin{tabular}{llllll}
\hline$T_{0}(\mathrm{~K})$ & $\lambda\left(\mathrm{Wm}^{-1} \mathrm{~K}^{-1}\right)$ & $\dot{\gamma}_{0}\left(\mathrm{~s}^{-1}\right)$ & $\tau_{0}(\mathrm{MPa})$ & $\beta$ & $m$ \\
\hline 300 & 54 & 1000 & 500 & 0.33 & 0.012 \\
\hline
\end{tabular}

Nevertheless in the following, $h_{V}$ and $h_{T}$ will be referred to as shear band widths.

A penalty method is introduced to fulfill the condition $h_{V}=h_{T}$. At this stage, $h_{V}$ and $h_{T}$ are the unknowns. The optimization condition (31) is then replaced by the new optimization problem:

$\operatorname{stat}_{h_{V}, h_{T}}\left[\Phi+\frac{1}{2} K\left(h_{V}-h_{T}\right)^{2}\right]$

where $K$ is a penalty coefficient. Note that for the proposed material flow law and the trial function, the stationarity of (33) with respect to $h_{V}$ is always satisfied whatever the value of $h_{V}$. The stationarity of (33) with respect to $h_{T}$ will lead to a nonlinear equation for $h_{T}$. After some mathematical development, we can prove that the corresponding relationship defined by Leroy and Molinari (1992) is retrieved, see Appendix A. As a consequence, our approach is able to capture the exact solution.

\subsubsection{Shear band width and central temperature}

The trial function adopted in the previous section for the temperature involved material parameters $m$ and $\beta$ which are related to the flow law. To avoid this drawback, a new set of variables is adopted to define velocity and temperature in the shear band. Instead of $h_{T}$ and $h_{V}$, we introduce $h$ and $T_{\max }$, so that the two trial functions become:

$V(y)=V_{0} \frac{\tanh (y / h)}{\tanh (H / h)}$,
$T(y)=T_{\max }-\left(T_{\max }-T_{0}\right) \frac{\ln (\cosh (y / h))}{\ln (\cosh (H / h))}$

where $h$ and $T_{\max }$ represent the shear band width and central temperature. The values of $T_{\max }$ and $h$ are determined by a stationary condition on $\Phi$ :

$\operatorname{stat}_{h, T_{\max }} \Phi\left(h, T_{\max }\right)$

Indeed, the stationarity of $\Phi$ with respect to $T_{\max }$ leads to an equation which represents energy conservation while the stationarity of $\Phi$ with respect to $h$ leads to an equation representing the momentum conservation. Fig. 4 represents the variation of $\Phi$ with respect to parameters $h$ and $T_{\max }$. Results are presented with $H=1.25 \mathrm{~mm}$ and $V_{0}=0.01108 \mathrm{~m} / \mathrm{s}$. Material parameters are those of Table 3. The solution of the thermo-mechanical coupled problem is then obtained through an optimization problem where one has to locate the saddle point.

Note that parameters $h$ and $T_{\max }$ are unknowns of our optimization problem, which serve to define continuous profiles of velocity and temperature. While the interpretation of $T_{\max }$ as the maximal temperature, in the middle of the ASB, is very clear, the interpretation of $h$ offers more freedom. Indeed, it is interesting to mention that there is no unique definition of the shear band width. For instance, Batra and Ko (1992) considered that the localized zone is fully formed when the shear stress drops to $85 \%$ of the peak value. In our approach, $h$ measures the distance where rapid variations of velocity and temperature are observed (see Fig. 5), and we identify this length with the shear band width. Alternatively, since the slab width $H$ is large compared to the shear band width $h$, then from relations (34), it is possible to derive a simple definition of $h$ : 


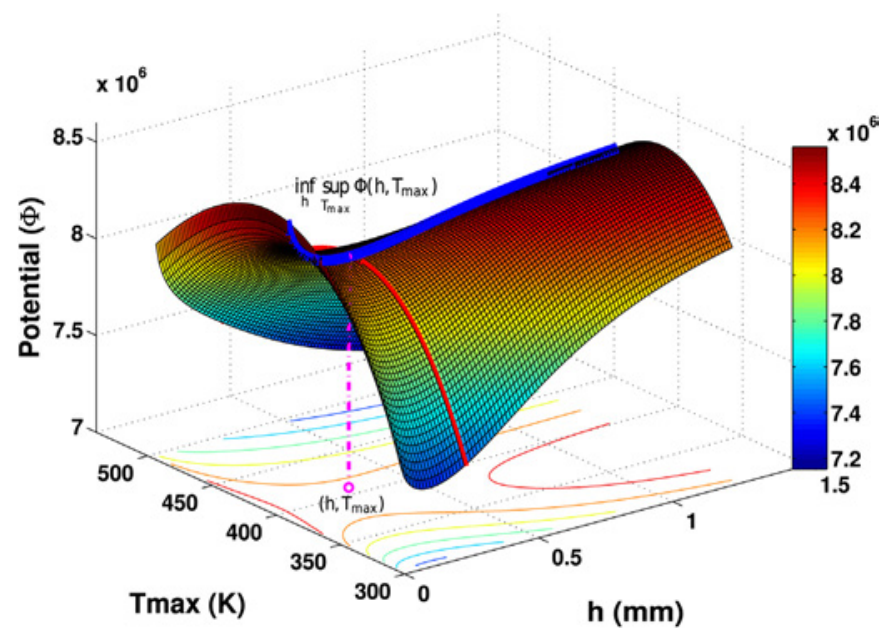

Fig. 4. Modeling of adiabatic shear band by the energy-based variational approach. The solution of the problem is located on the saddle point.

$V(h)=V_{0} \tan \mathrm{h}(1)$

i.e. the distance from the center at which the velocity reaches $V_{0} \tan \mathrm{h}(1)$.

\subsection{Results and analysis}

First, predictions based on the two approaches (set of variables $\left(h_{V}, h_{T}\right)$ or $\left.\left(h, T_{\max }\right)\right)$ are compared. Due to the strong non-linearity of the Euler-Lagrange equations (31), an optimized algorithm of the Newton-Raphson method named the trust-region method, is adopted to solve the set of stationary problem (35). In addition, the equilibrium temperature profile $\Theta(y)$ at a given iteration is taken as the (external) temperature profile obtained at the previous iteration. Table 4 shows the numerical solutions $\left(\bar{h}_{V}=\bar{h}_{T}\right)$ and $\left(\bar{h}, T_{\max }\right)$ obtained for different strain rate sensitivities, where $\bar{h}_{V}=h_{V} / H$ and $\bar{h}=h / H$. It is shown that analytical results of Leroy and Molinari (1992) are found. Fig. 5 presents the profiles of velocity and temperature in the slab for the reference set of parameters presented in Table 3 and for $H=1.25 \mathrm{~mm}, V_{0}=0.1 \mathrm{~m} / \mathrm{s}$. The shear band thickness is $0.08965 \mathrm{~mm}$ and the central temperature is $893.3 \mathrm{~K}$.

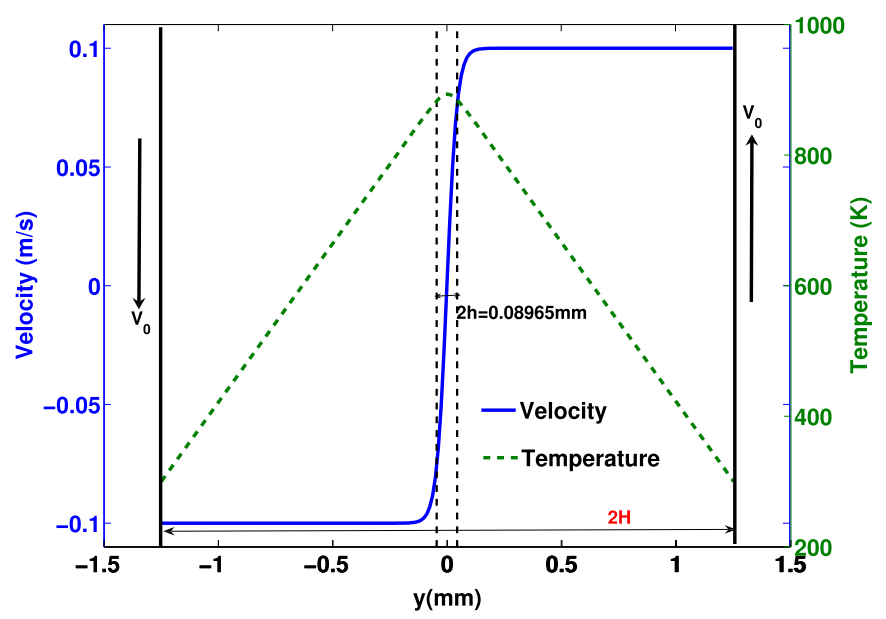

Fig. 5. Profiles of velocity and temperature in steady state. The formation of ASB is considered and exact results of Leroy and Molinari (1992) are retrieved (The nominal strain rate is $90 \mathrm{~s}^{-1}$, but the average strain rate inside the band is $2230 \mathrm{~s}^{-1}$ ).
Table 4

Shear band width (dimensionless) and central temperature $\left(\beta=0.38 ; V_{0}=0.1108 \mathrm{~m} /\right.$ s).

\begin{tabular}{|c|c|c|c|c|c|}
\hline \multirow[b]{3}{*}{$m=0.012$} & \multicolumn{3}{|c|}{ Variational model } & \multicolumn{2}{|c|}{ Analytical solutions } \\
\hline & \multirow{2}{*}{$\begin{array}{l}\bar{h}_{V} \\
0.0310\end{array}$} & \multicolumn{2}{|c|}{$\left(\bar{h} \quad T_{\max }[K]\right)$} & \multicolumn{2}{|c|}{$\left.\overline{(\bar{h}} \quad T_{\max }[K]\right)$} \\
\hline & & $(0.0311$ & $896.6)$ & $(0.0311$ & $896.6)$ \\
\hline$m=0.06$ & 0.1535 & ( 0.1535 & $851.3)$ & ( 0.1535 & $851.3)$ \\
\hline$m=0.12$ & 0.3126 & ( 0.3126 & $775.0)$ & ( 0.3126 & $775.0)$ \\
\hline
\end{tabular}

In the following, we adopt the set of variables $h$ and $T_{\max }$ to describe the shear localization. Fig. 6(a) illustrates the influence of the imposed velocity $V_{0}$ on $h$ and $T_{\max }$. The nominal strain rate is varying up to $1600 \mathrm{~s}^{-1}$ for a velocity of $2 \mathrm{~m} / \mathrm{s}$ prescribed at the frontier of the domain. When the velocity increases, the shear band width $h$ decreases and the central temperature in the band increases. From this figure, we observe that the reduction of the shear band width slows down when $V_{0}>0.5 \mathrm{~m} / \mathrm{s}$. The occurrence of the adiabatic shear band is the result of a thermo-mechanical instability. In a thermal softening material, a higher strain rate causes a smaller band width and also brings more dissipation and heat generation in the band. As the strain rate increases, the time scale of the deformation process (which is proportional to the inverse of the strain rate) becomes smaller so the heat removal by conduction is less efficient, leading to a higher central temperature.

The influence of thermal conductivity $\lambda$ on $h$ and $T_{\max }$ is also analyzed in Fig. 6(b). The shear band width increases almost linearly with $\lambda$, while the central temperature decreases in a nonlinear way. Numerical analysis also illustrates the well known fact that adiabatic shear band is a misnomer, since heat conduction plays an important role in the final process of ASB formation, significantly affecting localization zone width and central temperature.

The shear band width and temperature computed in our model are calculated under steady state assumption, when strain hardening is saturated. Note that in experiments, fracture of the specimen probably occurs before a steady state is reached. Leroy and Molinari (1992) discussed that point and concluded that shear bands observed in torsional Kolsky bar test are not tending towards a steady state. Therefore, our approach does not provide direct comparison with experimental results. Nevertheless, it is worth mentioning that shear band widths captured by our approach are reasonable when compared to results of the literature. Typical shear band width are in the range $10-100 \mu \mathrm{m}$ for a wide range of materials. For instance, Marchand and Duffy (1988) measured shear band width of $20 \mu \mathrm{m}$ for a HY100 steel (nominal strain rate of $1600 \mathrm{~s}^{-1}$ ). In our approach, with the proposed definition of the shear band width, the corresponding shear band width is around $10 \mu \mathrm{m}$.

Note also that central temperatures obtained within the band are larger than in experiments (Marchand and Duffy, 1988; Guduru et al., 2001). First, one has to recognize that the Taylor-Quinney coefficient is set to one (all plastic work is transformed into heat), so with a lower value (as measured in experiments), the temperature increase would be lower. Second, the temperature obtained is the one which exists under steady state, when the hardening is saturated. Note that in experiments, this regime is never reached and the specimen is fractured before. Finally, temperature measurements are seldom provided in the literature. Marchand and Duffy (1988) have provided some measurements which correspond to an average temperature measured on the surface by a device with a spot size and not within the material itself.

\section{Extension to various constitutive laws}

The shapes of the trial functions (32) were defined based on analytical expressions obtained by Leroy and Molinari (1992). 

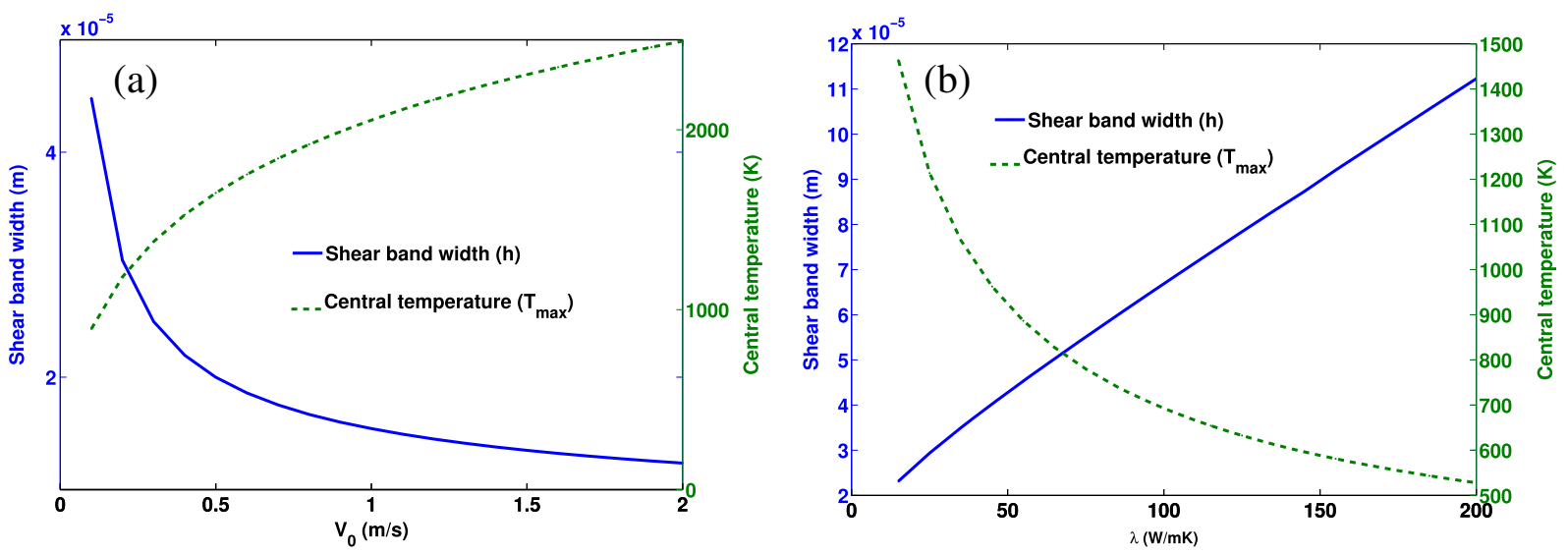

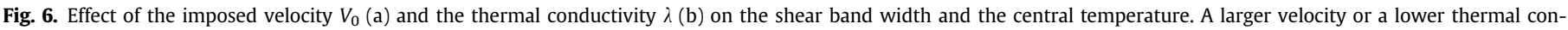
ductivity increases the central temperature and reduces the shear band width.

These expressions were exact when considering the material flow law (28) but depending upon material parameters ( $m$ and $\beta$ ). To extend this formalism, we have defined a new set of functions (34) depending on parameters $\left(h, T_{\max }\right)$ which characterizes the shear localization independently of material behavior. In this section, we propose to validate our approach by considering two different constitutive models: power law and Johnson-Cook model. Trial functions (34) are used and predictions of the variational approach are compared to FEM solutions. The latter are obtained by applying the FEM formulation sketched in Appendix C.

\subsection{Power law}

In this constitutive model, rate dependence and thermal softening are described via power law expressions:

$\tau=\tau_{0}\left(\frac{T}{T_{0}}\right)^{\nu}\left(\frac{\dot{\bar{\gamma}}^{p}}{\dot{\gamma}_{0}}\right)^{m}$

where $\dot{\bar{\gamma}}^{p}$ is the equivalent plastic shear strain rate, and where $\tau_{0}$ represents the flow stress level for $T=T_{0}$ and $\dot{\bar{\gamma}}^{p}=\dot{\gamma}_{0}$. Constitutive parameters $\dot{\gamma}_{0}$ and $T_{0}$ are thus reference strain rate and temperature. Exponent $\nu$ defines the thermal softening. Under steady state, $\dot{\gamma}^{e}=0$, and thus $\dot{\bar{\gamma}}^{p}=|\dot{\gamma}|$. Assuming the material obeys a von Mises law, and under simple shear, we obtain:

$\dot{\bar{\gamma}}^{p}=\left|V_{, y}\right|$

By adopting the following dissipation potential:

$\psi^{*}\left(\dot{\bar{\gamma}}^{p} ; \Theta\right)=\frac{\tau_{0} \dot{\gamma}_{0}}{m+1}\left(\frac{\Theta}{T_{0}}\right)^{\nu}\left(\frac{\dot{\bar{\gamma}}^{p}}{\dot{\gamma}_{0}}\right)^{m+1} \quad m \in[0,1]$

and using the canonical formulations for the velocity and temperature (34) in variational principle (35), Euler-Lagrange equations for the unknowns $h$ and $T_{\max }$ are defined. The adiabatic shear band is then fully characterized by solving two nonlinear equations.

For illustrative purpose, the material considered here is representative of a CRS 1018 steel, with constitutive parameters given in Table 5.

Table 5

Material parameters for CRS 1018 steel from Leroy and Molinari (1992).

\begin{tabular}{llllll}
\hline$\nu$ & $\tau_{0}(\mathrm{MPa})$ & $\lambda\left(\mathrm{Wm}^{-1} \mathrm{~K}^{-1}\right)$ & $m$ & $\dot{\gamma}_{0}\left(\mathrm{~s}^{-1}\right)$ & $T_{0}(\mathrm{~K})$ \\
\hline-0.38 & 500 & 54 & 0.012 & 1000 & 300 \\
\hline
\end{tabular}

For validation, finite element calculations based on the incremental variational approach (Yang et al., 2006) are used to determine the evolution of the velocity and the temperature in the shear layer. In contrast with traditional methods for solving thermo-mechanical problems, the tangent matrix of our FEM model is symmetric, as a result of the variational structure of the formulation. The material flow law adopted in FEM is (37) and elasticity is accounted for in the numerical simulation. Due to large strain rate in the localization zone, the mesh is dense in the vicinity of the layer's center.

Fig. 7 provides the time evolution of velocity and temperature when $V_{0}=0.1108 \mathrm{~m} / \mathrm{s}$ and $H=1.25 \mathrm{~mm}$. Note that Fig. 7 presents the results for $y<H$ (half of the layer). At the beginning of the evolution, the loading is homogeneous and the velocity profile is linear, as expected. Steady state is reached asymptotically when time increases. In that case, the velocity field is evolving significantly in a narrow band and is nearly constant outside. Temperature significantly evolves as well: from a homogeneous initial stage, the temperature increases and reaches a maximum in the central part of the layer when adiabatic shear band has formed.

In order to compare FEM and the proposed model, the previous definition of $h$ (36) is also adopted to interpret FEM results. Fig. 8 shows the time evolution of shear band width calculated by FEM. As time evolves, the calculated shear band width decreases and saturates for $t>0.15 \mathrm{~s}$. Fig. 9 compares the profiles of velocity and temperature under steady state obtained via FEM and the proposed model. Clearly, there is an excellent agreement between the two approaches. Almost no difference exists between results. Table 6 gives the shear band width and the central temperature provided by the FEM and the variational method when $V_{0}=0.1108 \mathrm{~m} / \mathrm{s}$ and $H=1.25 \mathrm{~mm}$. Thus, this first test proves the efficiency of the proposed approach.

When comparing results of Tables 4 and 6 , for the same configuration (velocity and band width), it is observed that the predicted shear band width is more than 3 times larger for CRS 1018 than for HY100. This trend is consistent with results of the literature. Indeed, in addition to HY100, Marchand and Duffy (1988) have performed testing for CRS 1018 and mentioned that the shear band is wider (around $100 \mu \mathrm{m}$ ). So clearly, even if our approach has not been built for direct comparison, the steady state results provided by our approach are consistent. An extension of the model to transient regime is necessary for a more precise comparison with results. Some of this work has been done by the authors (see Su, 2012), with promising perspectives, but a detailed description would go beyond the scope of this paper. Thus to validate our approach, and for salient comparisons (same material law and same configuration and same assumption of steady state), the recourse to FEM is necessary and compulsory. 


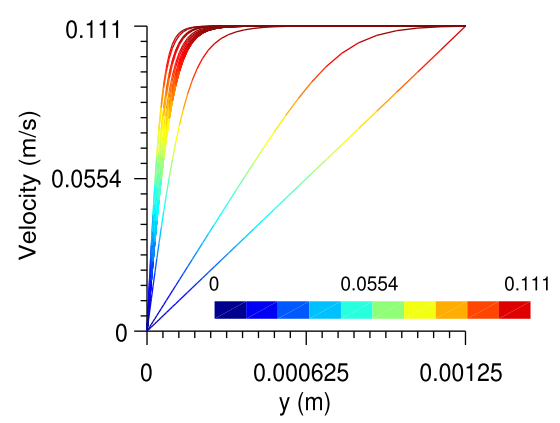

(a)

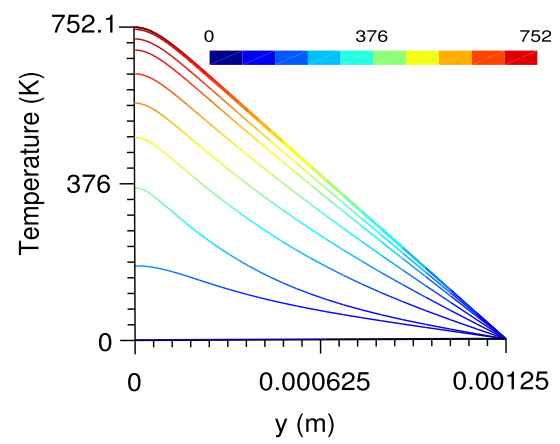

(b)

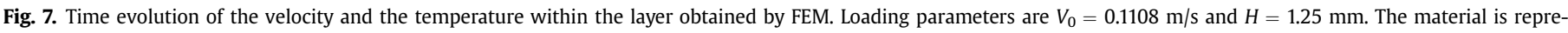
sentative of CRS 1018 steel, and the material behavior is described by a power law.

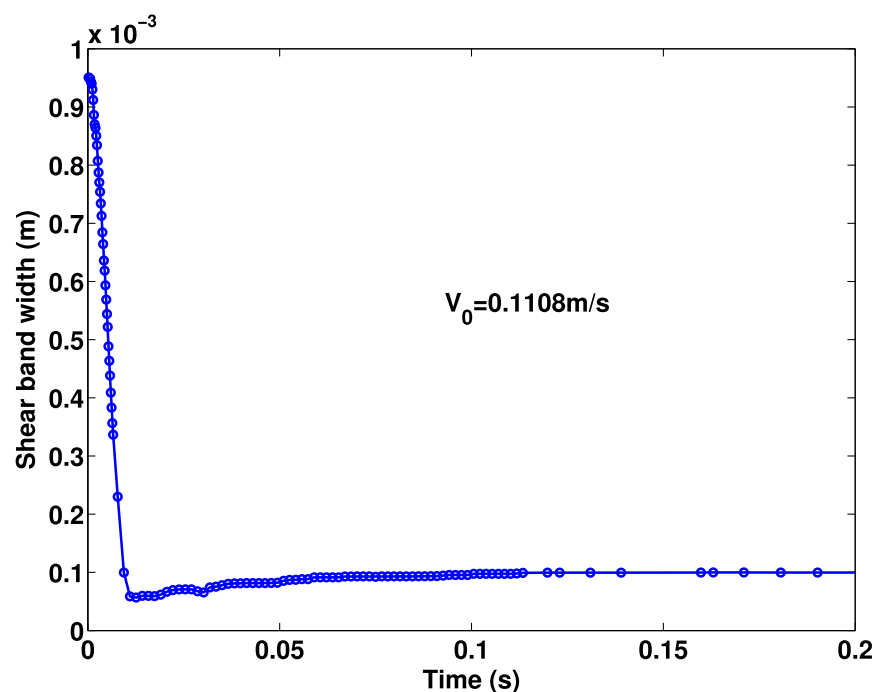

Fig. 8. Time evolution of shear band width obtained by FEM. Loading parameters are $V_{0}=0.1108 \mathrm{~m} / \mathrm{s}$ and $H=1.25 \mathrm{~mm}$. Material is representative of CRS 1018 steel, see Table 5 for material parameters.

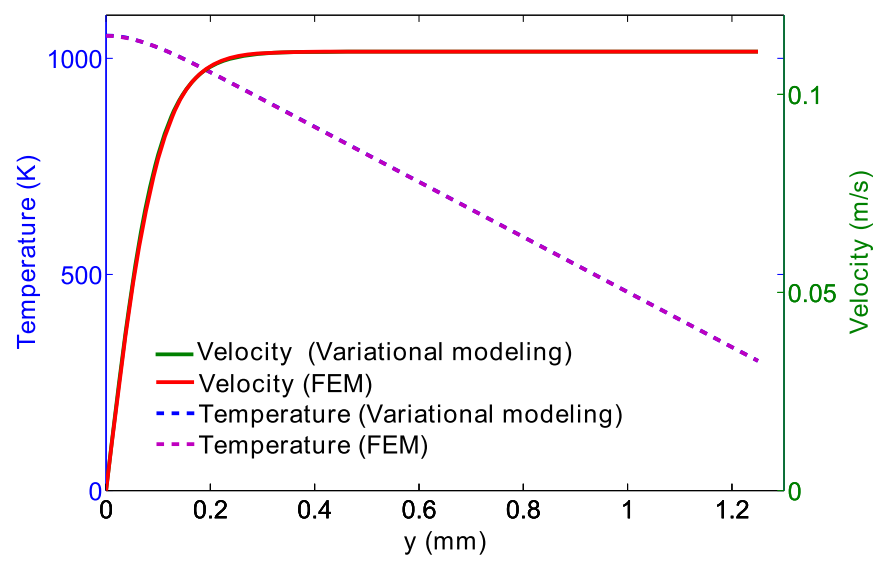

Fig. 9. Comparison of the velocity and temperature profiles in steady state obtained using the two approaches: FEM and variational model. Loading parameters are $V_{0}=0.1108 \mathrm{~m} / \mathrm{s}$ and $H=1.25 \mathrm{~mm}$.

\subsection{Johnson-Cook model}

When considering high strain rate loading, the Johnson-Cook (JC) model (Johnson and Cook, 1983) is widely adopted to represent rate dependent thermo-mechanical behavior of materials:

$\sigma_{y}\left(\dot{\bar{\varepsilon}}^{P}, \bar{\varepsilon}^{P}, T\right)=\left(A+B\left(\bar{\varepsilon}^{p}\right)^{n}\right)\left[1+C \log \left(\frac{\dot{\bar{\varepsilon}}^{p}}{\dot{\varepsilon}_{0}}\right)\right]\left(1-T^{* q}\right)$

where the non-dimensional temperature $T^{*}$ is defined as:

$T^{*}= \begin{cases}0 & \text { if } T<T_{0} \\ \frac{T-T_{0}}{T_{m}-T_{0}} & \text { if } T_{0}<T<T_{m} \\ 1 & \text { if } T>T_{m}\end{cases}$

Parameters $A, B, c, n$ and $q$ are material constants, $\dot{\varepsilon}_{0}$ is the reference effective strain rate, here chosen as $1.0, T_{m}$ is the melting temperature, and $T_{0}$ a reference temperature. To further test our modeling in the steady state, the hardening contribution in the JC model is disregarded by setting $B=0$. The corresponding dissipation potential is then given by:

$$
\begin{aligned}
\psi^{*}\left(\dot{\bar{\varepsilon}}^{p} ; \Theta\right)= & A_{d}\left(1-T^{*}(\Theta)^{q}\right) \dot{\bar{\varepsilon}}^{p}+A\left(1-T^{*}(\Theta)^{q}\right) \\
& \times C \dot{\varepsilon}_{0}\left[\frac{\dot{\bar{\varepsilon}}^{p}}{\dot{\varepsilon}_{0}} \log \left(\frac{\dot{\bar{\varepsilon}}^{p}}{\dot{\varepsilon}_{0}}\right)-\frac{\dot{\bar{\varepsilon}}^{p}}{\dot{\varepsilon}_{0}}+1\right]
\end{aligned}
$$

where $A_{d} \leq A$. The choice of $A_{d}$ affects the fraction of plastic energy transformed into heat. If $A_{d}=A$, the classical Taylor-Quinney coefficient is equal to unity. If $A_{d}<A$, this coefficient is lower than unity, and the total power density functional (29) must be modified by adding a $\dot{W}$ term in the integral, with

$\dot{W}\left(\dot{\bar{\varepsilon}}^{p}, \Theta\right)=\left(A-A_{d}\right)\left(1-T^{*}(\Theta)^{q}\right)^{p}$

Note that since we are focusing on the steady-state regime in this paper, where all hardening mechanisms are saturated, and thus probably all plastic energy storage mechanisms as well, this latter case may not be relevant here.

Table 6

Shear band width $h$ and central temperature $T_{\max }$ obtained by two approaches (analytical solutions and FEM).

\begin{tabular}{lll}
\hline & Variational modeling & FEM \\
\hline$h(\mathrm{~mm})$ & 0.0991 & 0.0997 \\
$T_{\max }(\mathrm{K})$ & 1052.8 & 1052.1 \\
\hline
\end{tabular}


Table 7

Material property for Ti-6Al-4V.

\begin{tabular}{llllllll}
\hline $\mathrm{A}(\mathrm{MPa})$ & $A_{d}(\mathrm{MPa})$ & $\mathrm{q}$ & $\lambda(\mathrm{W} / \mathrm{mK})$ & $\mathrm{C}$ & $\dot{\varepsilon}_{0}\left(\mathrm{~s}^{-1}\right)$ & $T_{0}(\mathrm{~K})$ & $T_{m}(\mathrm{~K})$ \\
\hline 792 & 792 & 1.03 & 54 & 0.014 & 1 & 300 & 1793 \\
\hline
\end{tabular}

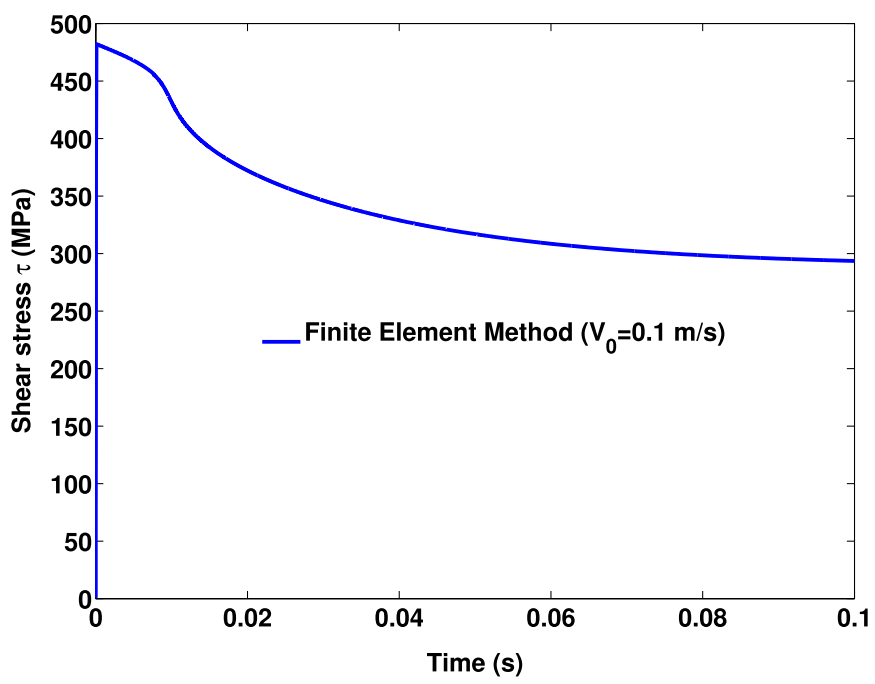

Fig. 10. Time evolution of the shear stress calculated by FEM $\left(V_{0}=0.1 \mathrm{~m} / \mathrm{s}\right.$ and $H=1.25 \mathrm{~mm}$ ). The material is representative of Ti-6Al-4V. The JC model has been considered.

For illustration's purpose, a layer made of Ti-6Al-4V is considered. Material parameters are given in Table 7. Fig. 10 presents the shear stress evolution within the band, as obtained by FEM when $H=1.25 \mathrm{~mm} ; V_{0}=0.1 \mathrm{~m} / \mathrm{s}$. The shear stress corresponds to the stress prevailing at the boundary $(y= \pm H)$. At first the material behaves elastically. When plasticity develops, the stress level decreases slightly due to a limited temperature evolution. Up to $t=0.01 \mathrm{~s}$, the deformation remains almost homogeneous. At a later stage, thermal softening induces strain localization and the stress drops rapidly. For $t>0.1 \mathrm{~s}$, a steady state develops. Meanwhile shear band width decreases with time and gradually evolves towards a defined value. Table 8 presents the values of the shear band width and central temperature for FEM and the variational model. Shear band width $h$ is well predicted while a small difference exists for the central temperature. The temperature is still increasing at $t=0.1 \mathrm{~s}$ for FEM, yet at a very reduced rate. We extrapolate that it will tend towards the limit value calculated by the variational model. Fig. 11 shows the stationary velocity and temperature profiles obtained by FEM and the variational model. A good agreement is found.

\section{Effect of thermal boundary conditions}

Slab width $H$ is a known parameter in the above energy-based variational modeling, and numerical analysis illustrates that ASB band width $h$ and central temperature $T_{\max }$ increase when $H$ is increased (Fig. 12), with isothermal boundary conditions $T=T_{0}$ and constant velocity $V=V_{0}$ enforced at $y=H$. As illustrated in Fig. 5, outside of the central zone of width $2 h$, velocity is almost constant

Table 8

Shear band width $h$ and central temperature $T_{\max }$ obtained by two approaches (JC Model). FEM values are measured at $t=0.1 \mathrm{~s}$.

\begin{tabular}{lcc}
\hline & Variational modeling & FEM \\
\hline$h(\mathrm{~mm})$ & 0.0421 & 0.0427 \\
$T_{\max }(\mathrm{K})$ & 952.8 & 938.7 \\
\hline
\end{tabular}

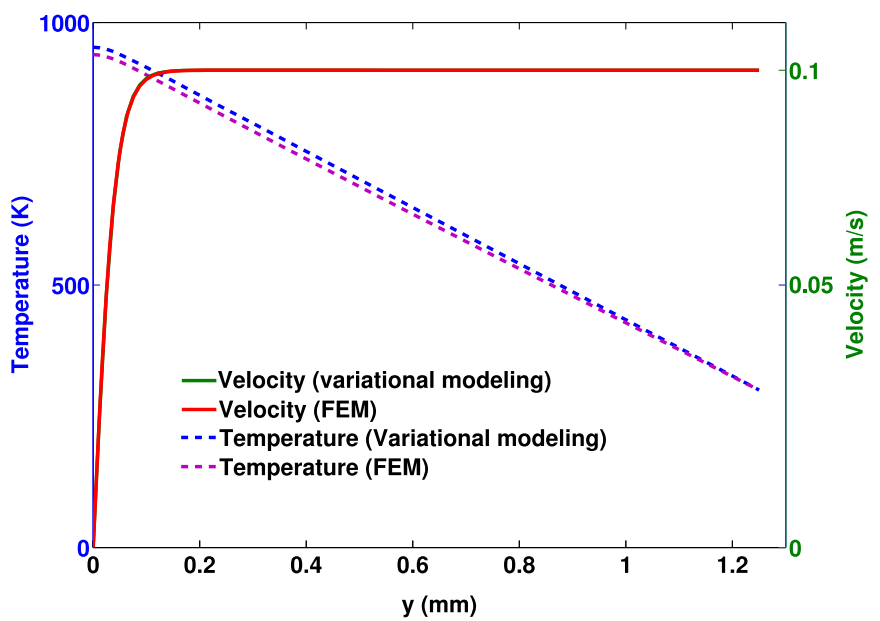

Fig. 11. Comparison of the velocity and temperature profiles in steady state obtained using the two approaches: FEM (at $t=0.1 \mathrm{~s}$ ) and variational model. Loading parameters are $V_{0}=0.1 \mathrm{~m} / \mathrm{s}$ and $H=1.25 \mathrm{~mm}$. The material is representative of Ti-6Al-4V. The flow law is described by the JC model (see Table 7 for material parameters).

while temperature varies linearly. From this observation, we can deduce that the effect of slab width $H$ on shear band width and central temperature is mostly linked to the imposition of a temperature $T_{0}$ at the boundary. Indeed, as shown in Fig. 13, increasing the width $H$ while maintaining a fixed temperature $T_{0}$ at the boundary leads to an overall increase of temperature, although the localization is slightly less marked (larger shear band width $h$ ). Isothermal boundary conditions thus result in a strong dependence of results on slab width $H$. We thus proceed to include mixed thermal boundary conditions, with the objective of limiting the direct dependency of results on slab width $H$.

The slab of width $2 \mathrm{H}$ is now embedded in a larger environment of constant temperature $T_{0}$. Heat removal from the sheared layer is supposed to exist via mixed boundary condition (similarly to the approach proposed in Leroy and Molinari (1992)):

$\mp \lambda \frac{\partial T}{\partial y}=c\left(T_{\text {ext }}-T_{0}\right)$ for $y= \pm H$

where $c$ is a film parameter (a.k.a. exchange coefficient). Isothermal boundary condition is retrieved for $c=+\infty$. Adiabatic condition is

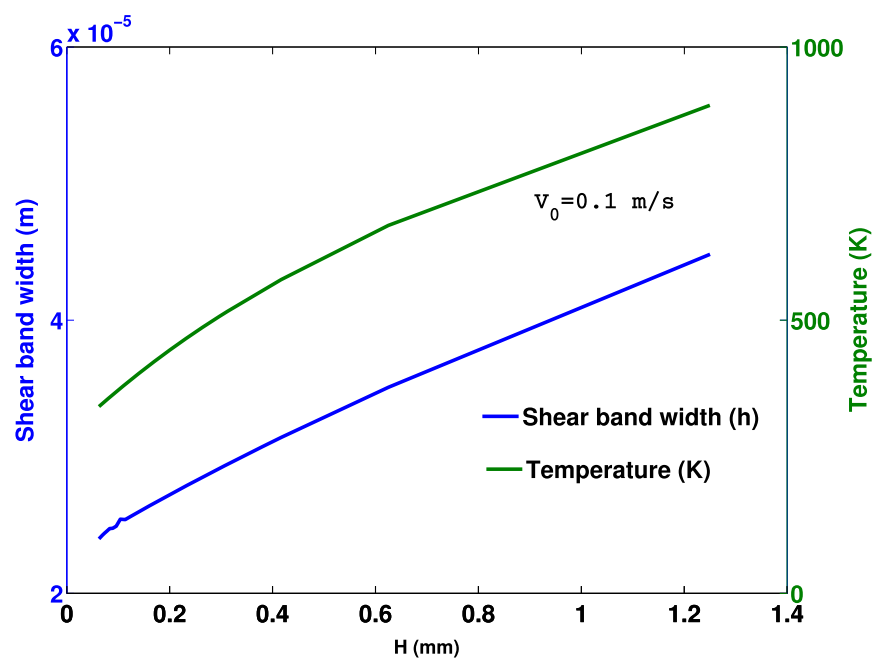

Fig. 12. Effect of the slab width on the shear band width and central temperature. The velocity is $0.1 \mathrm{~m} / \mathrm{s}$. The material, described by exponential law, is representative of HY100 steel. 

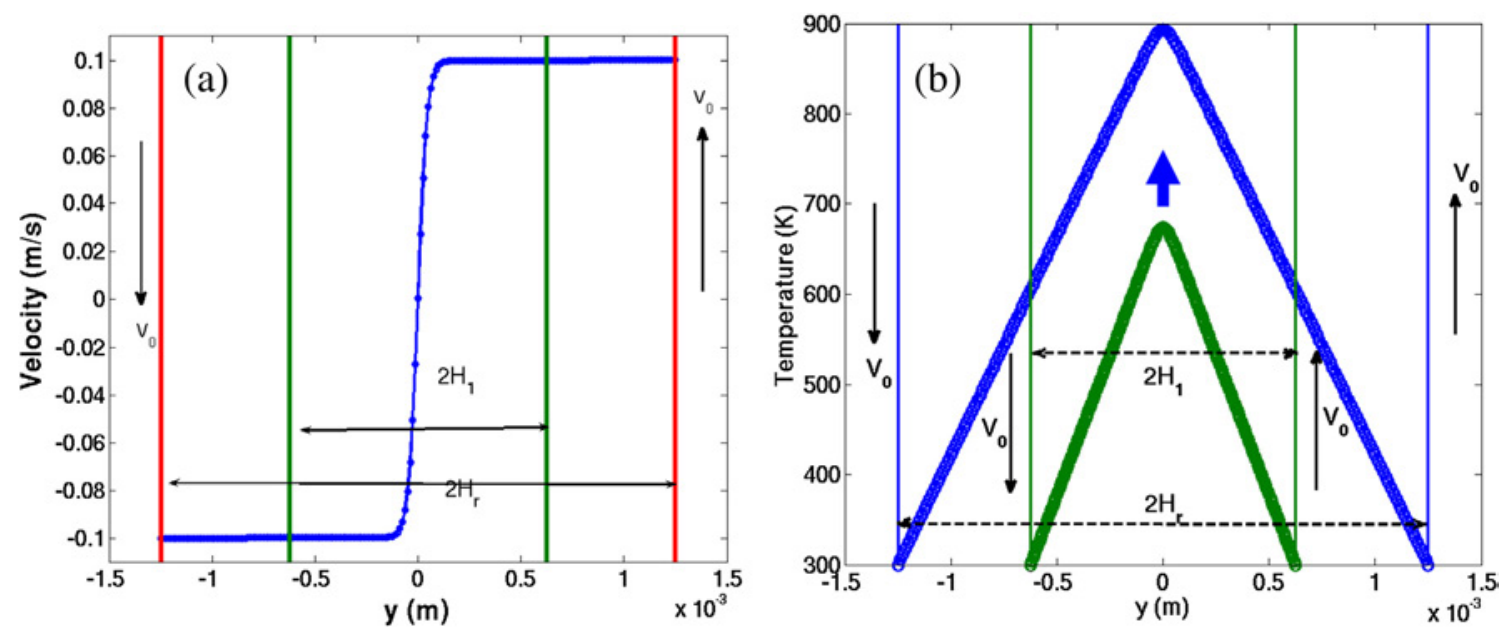

Fig. 13. Profiles of velocity (a) and temperature (b) in layers of different width.

obtained when $c=0$. Owing to the new boundary condition, $T( \pm H)$ is different from $T_{0}$ and we denote $T( \pm H)=T_{\text {ext. }}$ For the new problem, the trial velocity profile is unchanged:

$V(y)=V_{0} \frac{\tan \mathrm{h}(y / h)}{\tan \mathrm{h}(H / h)}$

while the trial temperature profile is now defined as:

$T(y)=T_{\max }-\left(T_{\max }-T_{\text {ext }}\right) \frac{\ln (\cos \mathrm{h}(y / h))}{\ln (\cos \mathrm{h}(H / h))}$

An expression of total power density potential accounting for mixed thermal boundary condition under steady-state conditions is given by:

$\Phi_{t}\left(h, T_{\max }, T_{\text {ext }}\right)=\int_{B} \Delta\left(\frac{T}{\Theta} \nabla \mathbf{V}, \frac{T}{\Theta} \dot{\mathbf{Z}},-\frac{\nabla T}{T} ; \Theta\right) \mathrm{d} V-2 \Phi_{\mathrm{tr}}$

with

$\Phi_{\mathrm{tr}}\left(T_{\text {ext }}\right)=\frac{1}{2} \frac{c}{\Theta_{1}}\left(T_{\text {ext }}-T_{0}\right)^{2}$

and $\Theta_{1}=\left.\Theta\right|_{y= \pm H}$. As before, balance equations can be obtained by optimization of $\Phi_{t}$ with respect to velocity and temperature. Additionally, mixed thermal boundary conditions are also obtained by the variation of $\Phi_{t}$ with respect to $T$ (see Appendix B). Using Ritz-Galerkin method, the variational modeling of an adiabatic shear band with heat exchange is then described by the following optimization problem:

$\underset{T_{\text {ext }}, h, T_{\max }}{\text { stat }} \Phi_{t}\left(h, T_{\max }, T_{\text {ext }}\right)$

Let us now consider the reference velocity and temperature profile $V_{r}(y)$ and $T_{r}(y)$ obtained from the parameter set: $H_{r}=1.25 \mathrm{~mm}, V_{0}=0.1 \mathrm{~m} / \mathrm{s}$ and $T_{0}=300 \mathrm{~K}$. In the following, $h_{r}$ and $T_{\text {max,r }}$ are denoted as the associated reference shear band width and reference maximum temperature. Considering a new problem with mixed thermal boundary condition in this part, we would like to evaluate the possibility of retrieving reference temperature and velocity profiles by adjusting the thermal boundary condition when varying the layer width $H$.

In contrast with conventional approaches where $c$ is considered as an input parameter, we will treat the heat exchange coefficient as an unknown parameter, which will be determined by imposing that we should recover similar solutions for various slab width $H$ (and a constant given loading velocity $V_{0}$ ). These profiles are characterized by $h=h_{r}$ and $T_{\max }=T_{\max , \mathrm{r}}$. We then look at solutions obtained for $h_{r}<H<H_{r}$, with mixed boundary conditions. In each case, we seek a value of exchange coefficient $c$ yielding a matching temperature profile for $-H \leq y \leq H$, as illustrated on Fig. 14. Since we want to obtain $T(y)=T_{r}(y)$, the sought value of $T_{\text {ext }}$ is defined by:

$T_{\text {ext }}=T_{\max , r}-\left(T_{\max , r}-T_{0}\right) \frac{\ln \left(\cosh \left(H / h_{r}\right)\right)}{\ln \left(\cosh \left(H_{r} / h_{r}\right)\right)}$

Adapting exchange coefficient $c$ to satisfy the above constraint for each value of $H$, shear band width and central temperature remain remarkably constant over a wide range of domain width, as shown in Fig. 15. In this figure, the relative norm for shear band width is defined by $h / h_{r}$. Relative norms for central temperature and shear stress are similarly defined. Unavoidably, there are some small fluctuations when $H$ comes close to the reference band width $h_{r}$, yet less than $5 \%$ in amplitude.

Fig. 16 presents the corresponding evolution of exchange coefficient $c$ with the slab width $H$. It appears from that figure that a linear relation exists between $\log \left(H_{r}-H\right)$ and $\log (c)$ :

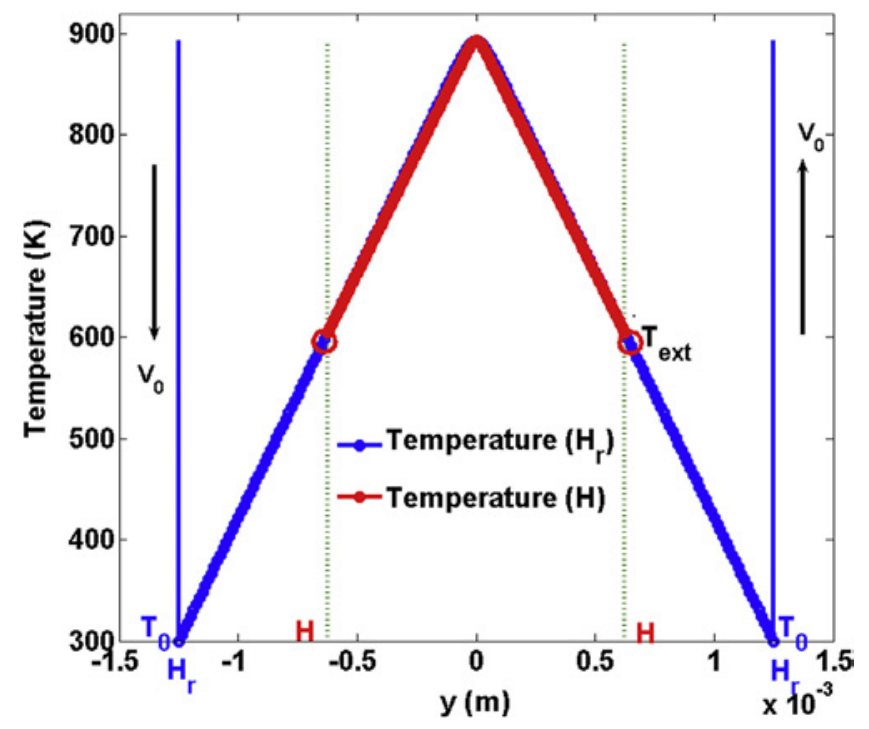

Fig. 14. Profiles of temperature on different structure sizes. 


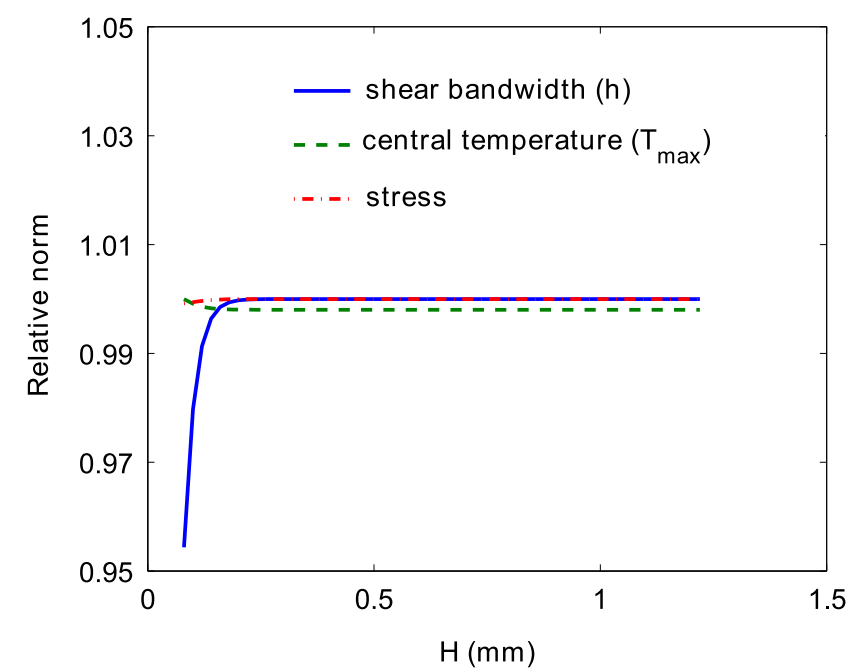

Fig. 15. Relative shear band width, central temperature and central shear stress for different structure sizes (with respect to the reference solution obtained with $H=1.25$ $\mathrm{mm}, V_{0}=0.1 \mathrm{~m} / \mathrm{s}$ )

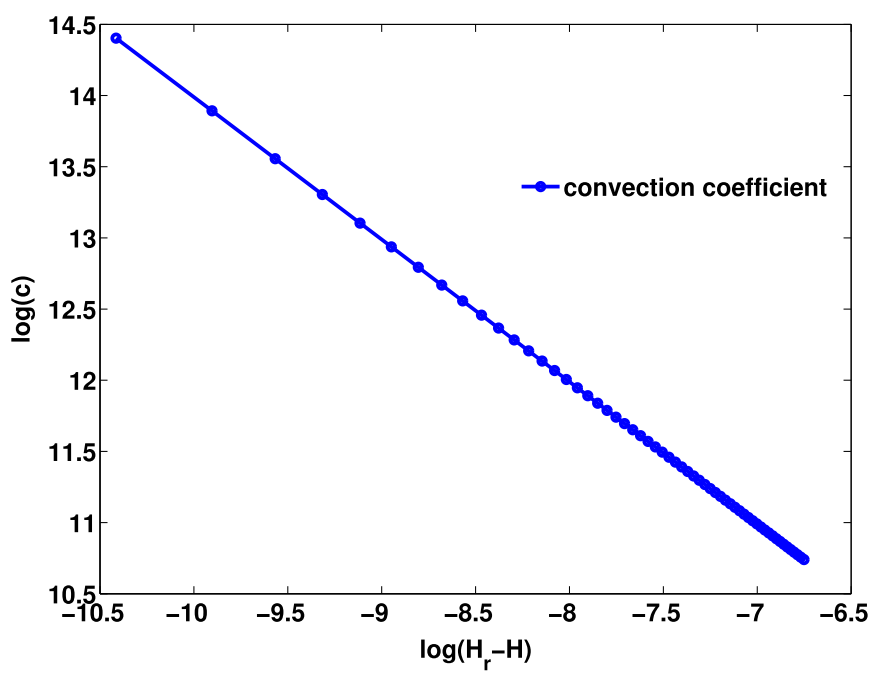

Fig. 16. Evolution of the Exchange coefficient as function of the width domain $H$. The material is representative of HY100 steel, and the material behavior is described by power law.

$\log (c)=a\left(V_{0}\right) \log \left(H_{r}-H\right)+b\left(V_{0}\right)$

with

$a\left(V_{0}\right)=-1, \quad b\left(V_{0}\right)=3.9889$ for $V_{0}=0.1 \mathrm{~m} / \mathrm{s}$

Calculations performed for different velocities $V_{0}$ have shown that the coefficient $a$ and $b$ do not vary with $V_{0}$. Note that expression (51) is limited to slab width $H$ such that $h_{r}<H<H_{r}$.

Expression (51) with parameter values (52) was successfully used in numerical simulations of transient evolution of velocity and temperature profiles (Su, 2012), showing that the above methodology actually allows to build a variational model of ASB formation.

\section{Conclusion}

An energy-based variational modeling of steady-state thermomechanical problems is proposed and successfully applied to solve classical problems such as Couette flow, thermal conduction in a layer and thermal Couette flow. Based on this approach, the formation of an adiabatic shear band in a slab subject to simple shear is investigated. The thermo-mechanical solution is obtained by means of an optimization problem. This continuum theory has proved its efficiency in deriving steady state solutions for ASB. For a specific constitutive model, the exact solution of Leroy and Molinari (1992) has been retrieved. Examples of application to more general constitutive laws are also proposed in the paper. By comparing to FEM, it is observed that steady state shear band width and maximum temperature are predicted with very good accuracy for various material descriptions (power law, Johnson Cook law). We also proposed a numerical formula of boundary exchange coefficient allowing to obtain consistent results for different slab widths.

The main advantage of the proposed method is numerical efficiency. The theory as presented here is restricted to the analysis of steady state regime while with FEM, the whole localization process from homogeneous strain to strain localization can be simulated. The analysis of transient regime can be analyzed with a variational principle similar to the one developed in this paper (Yang et al., 2006), but these results will be detailed elsewhere, see Su (2012) or Su and Stainier (2011) for more details. In all cases, the proposed approach is not aiming at representing the very early stages of shear band formation and propagation, which would require to model the ASB tip (Gioia and Ortiz, 1996; Mercier and Molinari, 1998).

Aside of the work on transient regime, further work could focus on exploiting this tool to explore the effect of material parameters and constitutive model characteristics on ASB features. In particular, it would be very interesting to assess the effect of variations in the Taylor-Quinney factor on ASB formation.

\section{Appendix A. Derivation of field equations from the variational principle}

The total power density potential (29) in Section 5 is formulated for coupled thermo-mechanical problems with rate and temperature dependent material behavior. Here it will be shown how the conservation equations of momentum and of energy are obtained by solving an optimization problem with respect to the velocity and the temperature. Taking the variation of (29) with respect to the velocity field, we obtain:

$$
\begin{aligned}
D_{V} \Phi(V, T)(\delta V)= & \int_{-H}^{H} \frac{\tau_{0}}{\left(\dot{\gamma}^{0}\right)^{m}} \exp \left[-\beta\left(\frac{\Theta}{T_{0}}-1\right)\right] \\
& \times\left(\frac{T}{\Theta}\right)^{m+1}\left(\frac{\partial V}{\partial y}\right)^{m} \frac{\partial \delta V}{\partial y} \mathrm{~d} y
\end{aligned}
$$

where $\delta V$ is a perturbation in velocity field satisfying $\delta V( \pm H)=0$. By integration by parts, the previous relation becomes:

This relation reduces to:

$D_{V} \Phi(V, T)(\delta V)=\left[\frac{\tau_{0}}{\left(\dot{\gamma}^{0}\right)^{m}} \exp \left[-\beta\left(\frac{\Theta}{T_{0}}-1\right)\right]\left(\frac{T}{\Theta}\right)^{m+1}\left(\frac{\partial V}{\partial y}\right)^{m} \delta V\right]_{-H}^{H}-\int_{-H}^{H} \frac{\partial}{\partial y}\left[\frac{\tau_{0}}{\left(\dot{\gamma}^{0}\right)^{m}} \exp \left[-\beta\left(\frac{\Theta}{T_{0}}-1\right)\right]\left(\frac{T}{\Theta}\right)^{m+1}\left(\frac{\partial V}{\partial y}\right)^{m}\right] \delta V \mathrm{~d} y$ 


$$
\begin{aligned}
D_{V} \Phi(V, T)(\delta V)= & -\int_{-H}^{H} \frac{\partial}{\partial y}\left[\frac{\tau_{0}}{\left(\dot{\gamma}^{0}\right)^{m}} \exp \left[-\beta\left(\frac{\Theta}{T_{0}}-1\right)\right]\left(\frac{T}{\Theta}\right)^{m+1}\right. \\
& \left.\times\left(\frac{\partial V}{\partial y}\right)^{m}\right] \delta V d y=0 \quad \forall \delta V
\end{aligned}
$$

Stationarity requires that this last relation holds for any $\delta V$. So one gets:

$\frac{\partial}{\partial y}\left[\frac{\tau_{0}}{\left(\dot{\gamma}^{0}\right)^{m}} \exp \left[-\beta\left(\frac{\Theta}{T_{0}}-1\right)\right]\left(\frac{T}{\Theta}\right)^{m+1}\left(\frac{\partial V}{\partial y}\right)^{m}\right]=0$

Since $\Theta=T$ and using the material flow stress definition (28), (A.4) reduces to:

$\frac{\partial \tau}{\partial y}=0$

The conservation of linear momentum is thus retrieved.

Next we treat the variation of (29) with respect to temperature:

$$
\begin{aligned}
& \frac{\tau_{0}}{\left(\dot{\gamma}^{0}\right)^{m}} \exp \left[-\beta\left(\frac{\Theta}{T_{0}}-1\right)\right]\left(\frac{\partial V}{\partial y}\right)^{m+1} \frac{1}{T}+\frac{\lambda}{T^{2}}\left(\frac{\partial T}{\partial y}\right)^{2} \\
& +\lambda \Theta \frac{\partial}{\partial y}\left(\frac{\partial T}{\partial y} \frac{1}{T^{2}}\right)=0
\end{aligned}
$$

After simplification, this equation provides conservation of energy:

$$
\frac{\tau_{0}}{\left(\dot{\gamma}^{0}\right)^{m}} \exp \left[-\beta\left(\frac{\Theta}{T_{0}}-1\right)\right]\left(\frac{\partial V}{\partial y}\right)^{m+1}+\lambda \frac{\partial^{2} T}{\partial y^{2}}=0
$$

\section{Appendix B. Heat equation with thermal boundary condition from the variational principle}

Extending the total potential in Appendix A, we consider the potential $\Phi_{t}$ with heat exchange on $y= \pm H$. Its form is recalled:

$$
\begin{aligned}
D_{T} \Phi(V, T)(\delta T)= & \int_{-H}^{H} \frac{\tau_{0}}{\left(\dot{\gamma}^{0}\right)^{m}} \exp \left[-\beta\left(\frac{\Theta}{T_{0}}-1\right)\right] \frac{T^{m}}{\Theta^{m+1}}\left(\frac{\partial V}{\partial y}\right)^{m+1} \delta T \mathrm{~d} y-\int_{-H}^{H} \lambda \frac{\Theta}{T^{2}} \frac{\partial T}{\partial y}\left(\frac{\partial \delta T}{\partial y}-\frac{\delta T}{T} \frac{\partial T}{\partial y}\right) \mathrm{d} y \\
= & \int_{-H}^{H} \frac{\tau_{0}}{\left(\dot{\gamma}^{0}\right)^{m}} \exp \left[-\beta\left(\frac{\Theta}{T_{0}}-1\right)\right] \frac{T^{m}}{\Theta^{m+1}}\left(\frac{\partial V}{\partial y}\right)^{m+1} \delta T \mathrm{~d} y+\int_{-H}^{H} \lambda \frac{\Theta}{T^{3}}\left(\frac{\partial T}{\partial y}\right)^{2} \delta T \mathrm{~d} y-\left[\lambda \frac{\Theta}{T^{2}} \frac{\partial T}{\partial y} \delta T\right]_{-H}^{H} \\
& +\int_{-H}^{H} \lambda \Theta \frac{\partial}{\partial y}\left(\frac{1}{T^{2}} \frac{\partial T}{\partial y}\right) \delta T \mathrm{~d} y=0 \quad \forall \delta T
\end{aligned}
$$

Since $\delta T( \pm H)=0, \Theta=T$ and previous equation should hold for any perturbation $\delta T$ of the temperature field at stationarity, we have:

$$
\begin{aligned}
\Phi_{t}= & \int_{B} \Delta\left(\frac{T}{\Theta} \nabla \mathbf{V}, \frac{T}{\Theta} \dot{\mathbf{z}},-\frac{\nabla T}{T} ; \Theta\right) \mathrm{d} V-\frac{1}{2} \frac{c}{\Theta_{-1}}\left(T(-H)-T_{0}\right)^{2} \\
& -\frac{1}{2} \frac{c}{\Theta_{1}}\left(T(H)-T_{0}\right)^{2}
\end{aligned}
$$

where $\Theta_{ \pm 1}=\left.\Theta\right|_{y= \pm H}$. The variation of $\Phi_{t}$ with respect to $T$ is:

$$
\begin{aligned}
D_{T} \Phi_{t}(V, T)(\delta T)= & \int_{-H}^{H} \frac{\tau_{0}}{\left(\dot{\gamma}^{0}\right)^{m}} \exp \left[-\beta\left(\frac{\Theta}{T_{0}}-1\right)\right] \frac{T^{m}}{\Theta^{m+1}}\left(\frac{\partial V}{\partial y}\right)^{m+1} \delta T \mathrm{~d} y-\int_{-H}^{H} \lambda \frac{\Theta}{T^{2}} \frac{\partial T}{\partial y}\left(\frac{\partial \delta T}{\partial y}-\frac{\delta T}{T} \frac{\partial T}{\partial y}\right) d y-\left.\frac{c}{\Theta_{-1}}\left[T(-H)-T_{0}\right] \delta T\right|_{y=-H} \\
& -\left.\frac{c}{\Theta_{1}}\left[T(H)-T_{0}\right] \delta T\right|_{y=H} \\
= & \int_{-H}^{H} \frac{\tau_{0}}{\left(\dot{\gamma}^{0}\right)^{m}} \exp \left[-\beta\left(\frac{\Theta}{T_{0}}-1\right)\right] \frac{T^{m}}{\Theta^{m+1}}\left(\frac{\partial V}{\partial y}\right)^{m+1} \delta T \mathrm{~d} y+\int_{-H}^{H} \lambda \frac{\Theta}{T^{3}}\left(\frac{\partial T}{\partial y}\right)^{2} \delta T \mathrm{~d} y-\left[\lambda \frac{\Theta}{T^{2}} \frac{\partial T}{\partial y} \delta T\right]_{-H}^{H}-\left.\frac{c}{\Theta_{-1}}\left[T(-H)-T_{0}\right] \delta T\right|_{y=-H} \\
& -\left.\frac{c}{\Theta_{1}}\left[T(H)-T_{0}\right] \delta T\right|_{y=H}+\int_{-H}^{H} \lambda \Theta \frac{\partial}{\partial y}\left(\frac{1}{T^{2}} \frac{\partial T}{\partial y}\right) \delta T \mathrm{~d} y=0 \quad \forall \delta T
\end{aligned}
$$


Since $\Theta=T$ and $\Theta_{ \pm 1}=\left.T\right|_{y= \pm H}=T_{\text {ext }}$, we then recover (A.8) once more, plus boundary conditions:

$$
\begin{aligned}
& \frac{\tau_{0}}{\left(\dot{\gamma}^{0}\right)^{m}} \exp \left[-\beta\left(\frac{\Theta}{T_{0}}-1\right)\right]\left(\frac{\partial V}{\partial y}\right)^{m+1}+\lambda \frac{\partial^{2} T}{\partial y^{2}}=0 \\
& -\lambda \frac{\partial T}{\partial y}=c\left(T_{\text {ext }}-T_{0}\right) \quad \text { on } y=H \\
& \lambda \frac{\partial T}{\partial y}=c\left(T_{\text {ext }}-T_{0}\right) \quad \text { on } y=-H
\end{aligned}
$$

\section{Appendix C. Finite element formulation of the unidimensional shear band problem}

A detailed description of the incremental variational formulation of general thermo-visco-plasticity can be found in Stainier (2013), for example. If we particularize to the case of the model shear band considered here, it reduces to

$$
\inf _{U_{n+1}} \sup _{T_{n+1}} \int_{-H}^{H}\left[\mathcal{W}\left(\gamma_{n+1}, T_{n+1}\right)-\Delta t \chi\left(-\frac{T_{n+1, y}}{T_{n+1}}\right)\right] \mathrm{d} y
$$

where

$$
\begin{aligned}
\mathcal{W}\left(\gamma_{n+1}, T_{n+1}\right)= & \inf _{\gamma_{n+1}^{p}}\left[W\left(\gamma_{n+1}, T_{n+1}, \gamma_{n+1}^{p}\right)-W\left(\gamma_{n}, T_{n}, \gamma_{n}^{p}\right)\right. \\
& \left.-\rho_{0} \eta_{n}\left(T_{n+1}-T_{n}\right)+\int_{t_{n}}^{t_{n+1}} \psi^{*}\left(\frac{T_{n+1}}{T_{n}} \frac{\Delta \gamma^{p}}{\Delta t} ; T(t)\right) \mathrm{d} t\right]
\end{aligned}
$$

with the shear strain $\gamma(y)=U_{y}(y)$. A time-discrete incremental finite element formulation can then be derived in a straightforward procedure.

Considering the symmetry of the sought solution, we will define the numerical model on the unidimensional domain $y \in[0, H]$, with boundary conditions

$$
U(0, t)=0, T_{, y}(0, t)=0 \text { and } U(H, t)=U_{0}\left(t_{n+1}\right), T(H, t)=T_{0}
$$

where $U_{0}(t)$ and $T_{0}$ are imposed displacement and temperature at the shear band boundary. We then introduce finite element type approximations of the displacement and temperature fields:

$$
\begin{aligned}
& U(y, t)=\sum_{a=1}^{N} U^{(a)}(t) N_{a}(y) \\
& T(y, t)=\sum_{a=1}^{N} T^{(a)}(t) N_{a}(y)
\end{aligned}
$$

where $U^{(a)}$ and $T^{(a)}$ are nodal displacements and temperatures, and $N_{a}(y)$ interpolating shape functions (i.e. with delta-Dirac properties). In practice, we have considered piecewise linear interpolation. Note also that we have chosen to use the same spatial discretization and shape functions for both displacement and temperature fields.
The variational principle (C.1) then becomes a nonlinear algebraic optimization problem:

$\inf _{\left\{U_{n+1}\right\}} \sup _{\left\{T_{n+1}\right\}} \Phi_{n}\left(\left\{U_{n+1}\right\},\left\{T_{n+1}\right\}\right)$

where $\quad\left\{U_{n+1}\right\}=\left\{U^{(1)}\left(t_{n+1}\right), \ldots, U^{(N)}\left(t_{n+1}\right)\right\} \quad$ and $\quad\left\{T_{n+1}\right\}=$ $\left\{T^{(1)}\left(t_{n+1}\right), \ldots, T^{(N)}\left(t_{n+1}\right)\right\}$ are arrays of nodal displacements and temperatures. This optimization problem, which has to be solved at each time step, can be solved by a standard Newton-Raphson procedure, complemented with a line-search to improve convergence.

\section{References}

Areias, P.M.A., Belytschko, T., 2007. Two-scale method for shear bands: thermal effects and variable bandwidth. Int. J. Numer. Meth. Eng. 72, 658-696.

Batra, R.C., Ko, K.I., 1992. An adaptive mesh refinement technique for the analysis of shear bands in plane strain compression of a thermoviscoplastic solid. Comput. Mech. 10 (6), 369-379.

DiLellio, J.A., Olmstead, W.E., 1997. Temporal evolution of shear band thickness. J. Mech. Phys. Solids 45 (3), 345-359.

DiLellio, J.A., Olmstead, W.E., 1998. Numerical solutions of shear localization in a finite slab. Mech. Mater. 29 (2), 71-80.

DiLellio, J.A., Olmstead, W.E., 2003. Numerical solution of shear localization in Johnson-Cook materials. Mech. Mater. 35, 571-580.

Dinzart, F., Molinari, A., 1998. Structure of adiabatic shear bands in thermoviscoplastic materials. Eur. J. Mech. A Solids 17, 923-938.

Dolinski, M., Rittel, D., Dorogoy, A., 2010. Modeling adiabatic shear failure from energy considerations. J. Mech. Phys. Solids 58 (11), 1759-1775.

Gioia, G., Ortiz, M., 1996. The two-dimensional structure of dynamic boundary layers and shear bands in thermoviscoplastic solids. J. Mech. Phys. Solids 44 (2), $251-292$.

Guduru, P.R., Rosakis, A.J., Ravichandran, G., 2001. Dynamic shear bands: an investigation using high speed optical and infrared diagnostics. Mech. Mater. 33 (7), 371-402.

Johnson, G.R., Cook, W.H., 1983. A constitutive model and data for metals subjected to large strains, high strain rates and high temperatures. In: Proceedings of the 7th International Symposium on Ballistics. The Hague, pp. 541-547.

Leroy, Y.M., Molinari, A., 1992. Stability of steady states in shear zones. J. Mech. Phys. Solids 40 (I), 181-212.

Marchand, A.. Duffy, J., 1988. An experimental study of the formation process of adiabatic shear bands in a structural steel. J. Mech. Phys. Solids 36 (3), 251-283.

Mercier, S., Molinari, A., 1998. Steady-State shear band propagation under dynamic conditions. J. Mech. Phys. Solids 46 (8), 1463-1495.

Merzer, A., 1982. Modelling of adiabatic shear band development from small imperfections. J. Mech. Phys. Solids 30 (5), 323-338.

Oliver, J., Cervera, M., Manzoli, O., 1999. Strong discontinuities and continuum plasticity models : the strong discontinuity approach. Int. J. Plast. 15, 319-351.

Ortiz, M., Leroy, Y., Needleman, A., 1987. A finite element method for localized failure analysis. Comput. Meth. Appl. Mech. Eng. 61, 189-214.

Rittel, D., Landau, P., Venkert, A., 2008. Dynamic recrystallization as a potential cause for adiabatic shear failure. Phys. Rev. Lett. 101 (16), 165501.

Stainier, L., 2013. A Variational Approach to Modeling Coupled Thermo-mechanical Nonlinear Dissipative Behaviors. In: Advances in Applied Mechanics, vol. 46. Academic Press, pp. 69-126 (Chapter 2).

Stainier, L., Ortiz, M., Mar. 2010. Study and validation of a variational theory of thermo-mechanical coupling in finite visco-plasticity. Int. J. Solids Struct. 47 (5), $705-715$.

Su, S., 2012. Energy-based Variational Variational Modeling of Adiabatic Shear Band Structure (Ph.D. thesis). Ecole Centrale de Nantes. http://tel.archives-ouvertes. fr/tel-00797178.

Su, S., Stainier, L., 2011. Energy-based variational modeling of adiabatic shear bands structure. In: Onate, E., Owen, D., Peric, D., Suárez, B. (Eds.), XI International Conference on Computational Plasticity. Fundamentals and Applications. In: http://congress.cimne.com/complas2011/proceedings/.

Wright, T.W., 2002. The Physics and Mathematics of Adiabatic Shear Band. In: Cambridge Monographs on Mechanics. Cambridge University Press.

Wright, T.W., Ockendon, H., 1992. A model for fully formed shear bands. J. Mech. Phys. Solids 40, 1217-1226.

Wright, T.W., Ravichandran, G., 1997. Canonical aspects of adiabatic shear bands. Int. J. Plast. 13 (4), 309-325.

Yang, Q., Mota, A., Ortiz, M., 2005. A class of variational strain-localization finite elements. Int. J. Numer. Meth. Eng. 62 (8), 1013-1037.

Yang, Q., Stainier, L., Ortiz, M., 2006. A variational formulation of the coupled thermo-mechanical boundary-value problem for general dissipative solids. J. Mech. Phys. Solids 54 (2), 401-424.

Zener, C., Hollomon, J.H., 1944. Effect of strain rate upon plastic flow of steel. J. Appl. Phys. 15 (1), 22-32. 